\title{
Inflammatory bowel disease patient's satisfaction with healthcare services received. Physicians' and nurses' perceptions
}

\author{
Francesc Casellas $^{1,4}$, Isabel Vera ${ }^{2}$, Daniel Ginard ${ }^{3}$ and Antonio Torrejón ${ }^{1,4}$, on behalf of the Grupo Español
} de Trabajo en Enfermedad de Crohn y Colitis Ulcerosa (GETECCU)

\author{
${ }^{1}$ Unitat Atenció Crohn-Colitis. Hospital Universitari Vall d'Hebron. Barcelona, Spain. ${ }^{2}$ Department of \\ Gastroenterology and Hepatology. Hospital Universitario Puerta de Hierro. Majadahonda, Madrid. Spain. \\ ${ }^{3}$ Hospital Universitari Son Espases. Palma de Mallorca, Spain. ${ }^{4}$ Centro de Investigación Biomédica en Red de \\ Enfermedades Hepáticas y Digestivas (CIBERehd)
}

\begin{abstract}
Background: patient satisfaction with healthcare services provided for inflammatory bowel diseases (IBD) is essential due to high resources use.

Objectives: the study aimed to describe patient satisfaction with healthcare services using the CACHE questionnaire and to assess gastroenterologist and nurse perception on patients' satisfaction.

Methods: observational multicentric prospective study in 35 Spanish hospitals. Patients included had Crohn's disease or ulcerative colitis. The study was approved by the Hospital Universitari Vall d'Hebron Ethics Committee. Scheduled study visits: baseline (patient sociodemographics and clinical data were collected), 2-4 and 6-months. Patient satisfaction with healthcare was assessed by CACHE questionnaire at each visit; it scores from 0-least satisfaction to 100-highest satisfaction.

Gastroenterologists and nurses answered once an adapted questionnaire.

Results: participating 290 patients (54.2\% males, 41.3 years old), 62 gastroenterologists and 47 nurses. At baseline mean (SD) CACHE score was 81.7 (10.9); satisfaction with clinician care was the highest, patient information the lowest. Scores did not change across study.

Gastroenterologist global score was 72.5 (9.8); Staff Care satisfaction was the highest, patient information the lowest. All scores were significantly lower than patients'.

Nurses' global score was 82.2 (8.5), clinician care satisfaction was the highest, centre facilities the lowest. Scores on satisfaction with clinician care, centre facilities, and patient information scored statistically lower than patients'.

No relationship was found between patients' satisfaction and patients characteristics.

Conclusions: IBD patients are satisfied with healthcare services provided, even though the information may be improved. Nurses'
\end{abstract}

Received: $03 / 04 / 2013$

Accepted: 19/08/2013

Correspondence: Francesc Casellas. Unitat Atenció Crohn-Colitis. Hospital Universitari Vall d'Hebron. Pg. Vall d'Hebron, 119. 08035 Barcelona, Spain e-mail: fcasellas@vhebron.net perception is similar to that of patients, physicians have a lower perception.

Key words: Crohn's disease. Ulcerative colitis. Satisfaction with healthcare. Physicians' perception. Nurses' perception.

\section{INTRODUCTION}

Chronic inflammatory bowel diseases (IBD) are common in Spain, the estimated prevalence for Crohn's disease (CD) is $87.5 / 100,000$ inhabitants (1) and for ulcerative colitis (UC) it is 110.0/100,000 inhabitants (1).

Because IBD is a complex chronic disorder, it requires a long-term management, with significant healthcare resource consumption (2). Patients require not only pharmaceutical treatment but also adequate healthcare facilities, access to diagnostic tests, and information regarding their disease, access to specialized staff (physicians, nurses and other healthcare staff). A positive patient-physician relationship is essential to optimize the quality of care and health outcomes in chronic diseases (3).

Patient satisfaction with the healthcare services provided is essential in IBD disease management (4). However, information relative to this topic is scarce. Thus, an observational study was conducted to develop and validate a new instrument for Spanish IBD patients (5), the CACHE questionnaire, in order to have a tool to measure patient satisfaction with global healthcare services.
Casellas F, Vera I, Ginard D, Torrejón A. Inflammatory bowel disease patient's satisfaction with healthcare services received. Physicians' and nurses' perceptions. Rev Esp Enferm Dig 2013;105:385-391. 
The objective of this paper is to describe patient satisfaction with the healthcare services showing the results obtained during the validation of the CACHE questionnaire and to identify those areas that could be improved. In addition, patient satisfaction is also compared to physician's and nurse's perception of patient satisfaction.

\section{METHODS}

An observational multicentric prospective study including IBD patients was conducted between May 2010 and March 2011, in 35 hospital centres from Spain.

Patients attending to the outpatient clinic for IBD were included consecutively. The study was approved by the Ethics Committee of the Hospital Universitari Vall d'Hebron (Barcelona, Spain). All the patients provided written informed consent prior to their inclusion in the study.

Patients included in the study were at least 18 years old with a clinical diagnosis of IBD (CD or UC) of at least one year duration. They could be receiving, or have received, any treatment for IBD (including surgery for CD). Patients were excluded if they were unable to read and write in Spanish, had any concomitant illness considered to potentially affect the study results, had colectomized UC, or were already participating in a clinical trial or had done so in the three months prior to study initiation.

The study consisted of three scheduled visits: baseline (inclusion), a follow-up visit at two months and a final visit at 4-6 months from baseline. At the baseline visit, patient data collected were sociodemographic (age, gender, education level, living arrangements, and smoking habits), clinical data referred to comorbid diseases, and to IBD (date of diagnosis, type of IBD, disease activity and surgery undergone). Disease activity was graded according to Harvey-Bradshaw and Montreal classification for CD and to Mayo index without endoscopy and Montreal classification for UC.

Patient satisfaction with the healthcare services and treatment received was assessed by the CACHE questionnaire, which validation of its Spanish version had been recently published (5). This questionnaire contains 31 items with five response options each, from "Totally agree" to "Totally disagree" and assessing the satisfaction with healthcare services in six domains related to the patient's satisfaction with information received, healthcare staff attention, doctor attention, centre facilities, accessibility to the centre and received support. The overall and each domain scores are standardized to score in a range from 0 to 100 , the lowest score indicating the worst possible satisfaction with the healthcare services. The CACHE questionnaire was self-administered at the beginning of each study visit.

At the time of the first visit of the first patient in each centre, the gastroenterologist and the nurse provided their age, gender, and number of years of professional experience, they also answered an ad hoc adapted CACHE questionnaire assessing their general perception of patients' satisfaction with the services provided in their centre; they answered this questionnaire only once.

The sample size was calculated to validate the CACHE questionnaire, which was the main objective of the observational study. No specific sample size calculation was done for the purpose of this paper.

\section{Statistical analysis}

Analysis was carried out using the statistical software package SPSS version 15.0 for Windows. The statistical significance level was set at 0.05 .

A descriptive analysis of sociodemographic and clinical characteristics is presented. Comparisons between the CACHE patients' score and the physicians'/nurses' scores were conducted using the non-parametric Wilcoxon ranksum test. A linear regression model was performed in order to define those patient variables influencing patient satisfaction with healthcare services.

\section{RESULTS}

\section{Patients' characteristics}

Initially 329 patients were recruited, 290 were considered for the per protocol analysis, $147(50.7 \%)$ had CD and $143(49.3 \%)$ UC. Thirty nine patients were excluded because: Two did not fulfil the selection criteria and $37 \mathrm{did}$ not finish the follow-up as scheduled per protocol (15 lost to follow-up, 1 patient's decision, 1 physician decision, 3 medication adverse events prevented them from attending to the study visits, and 17 the reason was not known).

Patients' mean (SD) age at inclusion was 41.3 (13.2) years, $52.4 \%$ were males, $22.2 \%$ had university studies, $74.1 \%$ lived in an urban or metropolitan area $(>30,000$ inhabitants). A third of patients had at least one comorbid condition (most frequent were dyslipidaemia, $9.3 \%$, and hypertension, $9.0 \%$ ), $42.2 \%$ of patients had never smoked (Table I). The average (SD) time of IBD evolution was 8.9 (7.0) years and $34.9 \%$ of patients with CD had undergone surgery for their condition. At study inclusion, $67.2 \%$ of the patients were receiving Tumor necrosis factor (TNF) inhibitors, $46.2 \%$ other immunosuppressants, $44.5 \%$ aminosalicylic acid and derivatives, and $12.1 \%$ glucocorticoids.

As per the Harvey-Bradshaw index, $56.6 \%$ of CD patients were on remission at baseline. Meanwhile the proportion of patients in remission as per the Montreal and Mayo Clinic Score classifications of severity were $66.4 \%$ and $80.3 \%$ respectively (Table II). During the study follow-up, $11.7 \%$ of patients presented a flare and $2.8 \%$ of the study patients were hospitalized at least once. At the final visit $69.3 \%$ of $\mathrm{CD}$ patients were in remission, for patients with UC the remission achieved was $82.3 \%$ as per the Montreal classification and $89.4 \%$ as per the Mayo Clinic Score (Table II). 
Table I. Patient sociodemographic and clinical baseline characteristics

\begin{tabular}{|c|c|c|c|}
\hline Characteristics & $C D$ & UC & $I B D$ \\
\hline Patients; n (\%) & $147(50.7 \%)$ & $143(49.3 \%)$ & $290(100 \%)$ \\
\hline Age (years); mean (SD) & $39.5(13.1)$ & $43.2(13.0)$ & $41.3(13.2)$ \\
\hline Gender (males); n (\%) & $78(53.1 \%)$ & $73(51.8 \%)$ & $151(52.4 \%)$ \\
\hline $\begin{array}{l}\text { Education level; } n \text { (\%) } \\
\text { Primary or less } \\
\text { Secondary or higher }\end{array}$ & $\begin{array}{l}58(39.5 \%) \\
89(60.5 \%)\end{array}$ & $\begin{array}{l}57(39.9 \%) \\
84(58.7 \%)\end{array}$ & $\begin{array}{l}115(39.7 \%) \\
173(59.7 \%)\end{array}$ \\
\hline $\begin{array}{l}\text { Habitat; } n(\%) \\
\text { < } 30.000 \text { inhabitants; n (\%) } \\
\text { > } 30.000 \text { inhabitants; n (\%) }\end{array}$ & $\begin{array}{l}35(23.8 \%) \\
112(76.2 \%)\end{array}$ & $\begin{array}{l}38(26.6 \%) \\
103(72.0 \%)\end{array}$ & $\begin{array}{l}73(25.2 \%) \\
215(74.1 \%)\end{array}$ \\
\hline Non-smokers; n (\%) & $52(35.6 \%)$ & $70(49.0 \%)$ & $122(42.2 \%)$ \\
\hline Concomitant disease; n (\%) & $45(30.6 \%)$ & $45(31.5 \%)$ & $90(31.0 \%)$ \\
\hline Time since diagnosis (years); mean (SD) & $9.7(7.5)$ & $8.0(6.4)$ & $8.9(7.0)$ \\
\hline
\end{tabular}

CD: Crohn's disease. UC: Ulcerative colitis. SD: Standard deviation.

\section{Patient satisfaction with healthcare services}

The mean (SD) CACHE score reported by IBD patients at baseline was 81.7 (10.9), with 100 indicating maximum satisfaction with healthcare. The domain with the highest score was Clinician Care Satisfaction with 91.4 (9.7) points and the lowest score was for the Patient Information Satisfaction domain, with 68.5 (18.0) points (Table III). CACHE scores (global and by domains) did not change significantly across the study follow-up visits (Fig. 1).

\section{Physicians' and nurses' characteristics}

A total of 62 gastroenterologist and 47 nurses participated in the patients' perception satisfaction survey.

The average (SD) age of gastroenterologists was 42.1 (7.8) years, a $54.8 \%$ were males and they had a mean (SD) of 15.6 (8.2) years of experience as gastroenterologist. The participating nurses had a mean (SD) age of 44.6 (10.8) years, $75 \%$ were females and they had a mean (SD) of 19.1 (9.5) years of experience as a nurse.

\section{Physician and nurse perception of the patient satisfaction with healthcare services and comparison with patients' perception}

The mean (SD) gastroenterologist score was 72.5 (9.8) points, Staff Care Satisfaction being the domain with the highest score [74.1 (15.6) points] and the Patient Information Satisfaction the one with the lowest score [60.3 (18.3) points]. Global scores and all individual domain scores were statistically significantly lower than patients' scores (Table IV).

The nurses' mean global score for the adapted CACHE questionnaire was (SD) of 82.2 (8.5) points, with the highest

Table II. Disease activity at baseline and during study follow-up: Harvey-Bradshaw index for CD patients and Montreal classification and Mayo Clinic for UC patients

\begin{tabular}{|c|c|c|c|}
\hline & Baseline $n(\%)$ & Follow-up n (\%) & Final $n(\%)$ \\
\hline \multicolumn{4}{|c|}{ Harvey-Bradshaw index for $C D$} \\
\hline Remission & $82(56.6 \%)$ & $103(71.0 \%)$ & $97(69.3 \%)$ \\
\hline Flare & $63(43.4 \%)$ & $42(29.0 \%)$ & $43(30.7 \%)$ \\
\hline \multicolumn{4}{|c|}{ Montreal classification for UC } \\
\hline Remission & $95(66.4 \%)$ & $108(76.1 \%)$ & $116(82.3 \%)$ \\
\hline Mild & $36(25.2 \%)$ & $24(16.9 \%)$ & $16(11.3 \%)$ \\
\hline \multicolumn{4}{|c|}{ Mayo Clinic score for UC (without endoscopic assessment) } \\
\hline Remission & $114(80.3 \%)$ & $121(85.2 \%)$ & $126(89.4 \%)$ \\
\hline Flare & $28(19.7 \%)$ & $21(14.8 \%)$ & $15(10.6 \%)$ \\
\hline
\end{tabular}

Scores were not available for all study visits for all patients. 
Table III. CACHE satisfaction questionnaire score, global and by domains at baseline and final visits, by type of IBD

\begin{tabular}{|c|c|c|c|c|c|c|}
\hline \multirow[t]{2}{*}{ Domain } & \multicolumn{2}{|c|}{$C D$} & \multicolumn{2}{|c|}{ UC } & \multicolumn{2}{|c|}{$I B D$} \\
\hline & Baseline & Final & Baseline & Final & Baseline & Final \\
\hline Staff care & $85.5(12.2)$ & $85.2(12.7)$ & $87.0(11.4)$ & $85.6(11.5)$ & $86.2(11.8)$ & $85.4(12.1)$ \\
\hline Centre facilities & $81.2(16.1)$ & $80.7(15.4)$ & $80.47(16.2)$ & $79.2(15.6)$ & $80.9(16.1)$ & $80.0(15.5)$ \\
\hline Patient information & $69.1(17.3)$ & $71.6(16.9)$ & $67.9(18.8)$ & $70.8(17.8)$ & $68.5(18.0)$ & $71.2(17.3)$ \\
\hline Global score & $81.6(11.1)$ & $81.9(11.6)$ & $81.8(10.6)$ & $81.8(10.6)$ & 81.7 (10.9) & $81.9(11.1)$ \\
\hline
\end{tabular}

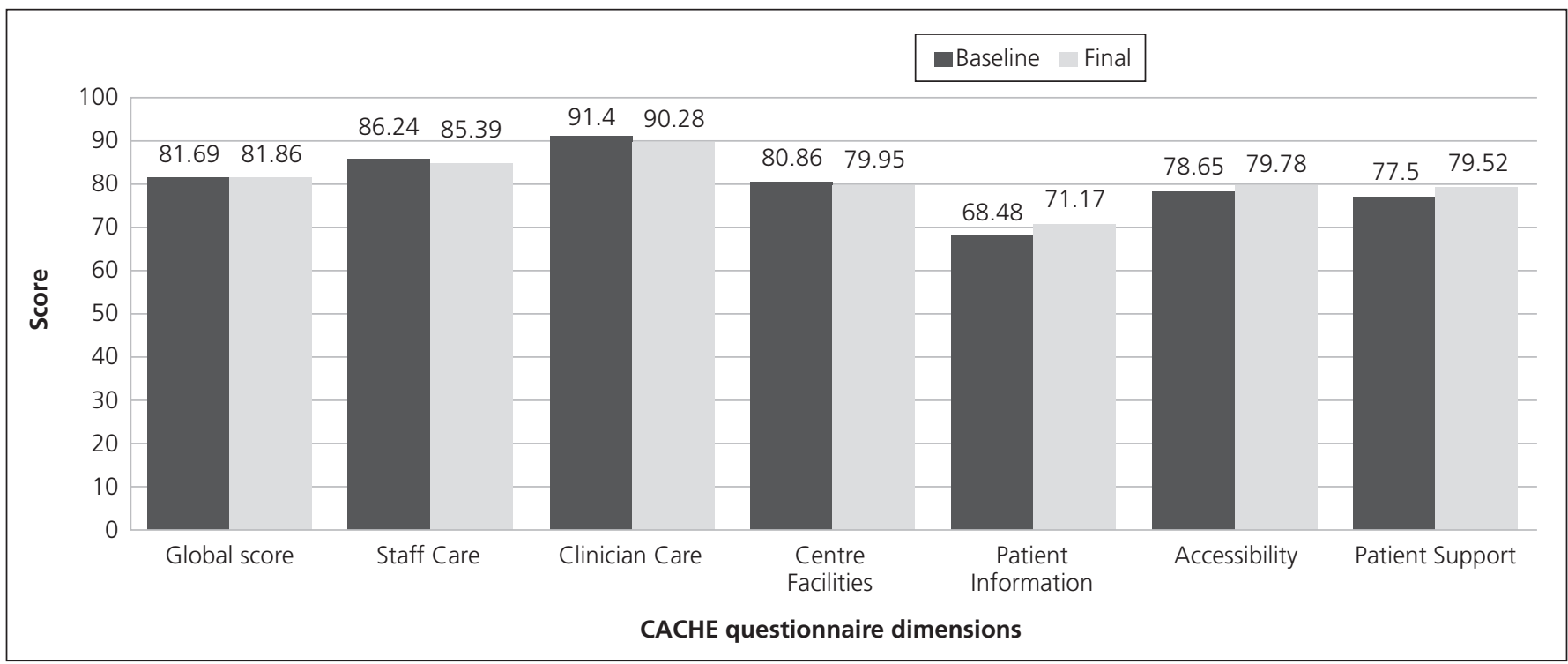

Fig. 1. CACHE questionnaire scores across the study.

score being Clinician Care Satisfaction [82.8 (23.4) points] and the lowest being Centre Facilities Satisfaction [69.5 (23.5) points]. Comparing nurses' scores with patients' scores, three domains were statistically lower in the nurses' responses; Clinician Care Satisfaction, Centre Care Facilities and Patient Information Satisfaction (Table IV, Fig. 2).

The bivariate regression found that socio-demographic and clinical variables had no relationship with patients'

Table IV. Comparison of the CACHE satisfaction questionnaire global and domain scores for gastroenterologists, nurses and patients at baseline

\begin{tabular}{|c|c|c|c|c|c|}
\hline \multirow[t]{2}{*}{ Domain } & \multirow{2}{*}{$\begin{array}{l}\text { Patients } \\
\text { Mean (SD) }\end{array}$} & \multicolumn{2}{|c|}{ Gastroenterologists } & \multicolumn{2}{|c|}{ Nurses } \\
\hline & & Mean (SD) & $p$-value* & Mean (SD) & $p$-value* \\
\hline Staff care & $86.2(11.8)$ & $74.1(15.6)$ & $<0.001$ & $80.4(23.4)$ & 0.264 \\
\hline Clinician care & $91.4(9.7)$ & $78.1(17.5)$ & $<0.001$ & $82.8(23.4)$ & 0.010 \\
\hline Centre facilities & $80.9(16.1)$ & $68.5(19.37)$ & $<0.001$ & $69.5(23.5)$ & 0.006 \\
\hline Patient information & $68.5(18.0)$ & $60.3(18.3)$ & 0.012 & $73.1(23.5)$ & 0.026 \\
\hline Accessibility & 78.7 (17.6) & $67.0(20.4)$ & 0.001 & $73.4(23.6)$ & 0.311 \\
\hline Patient support & 77.5 (17.3) & $67.7(20.3)$ & 0.008 & $76.3(23.7)$ & 0.709 \\
\hline Global score & 81.7 (10.9) & $72.5(9.8)$ & $<0.001$ & $82.2(8.5)$ & 0.934 \\
\hline
\end{tabular}

*Comparison vs. patients' score with the non-parametric Wilcoxon sum-rank test. 


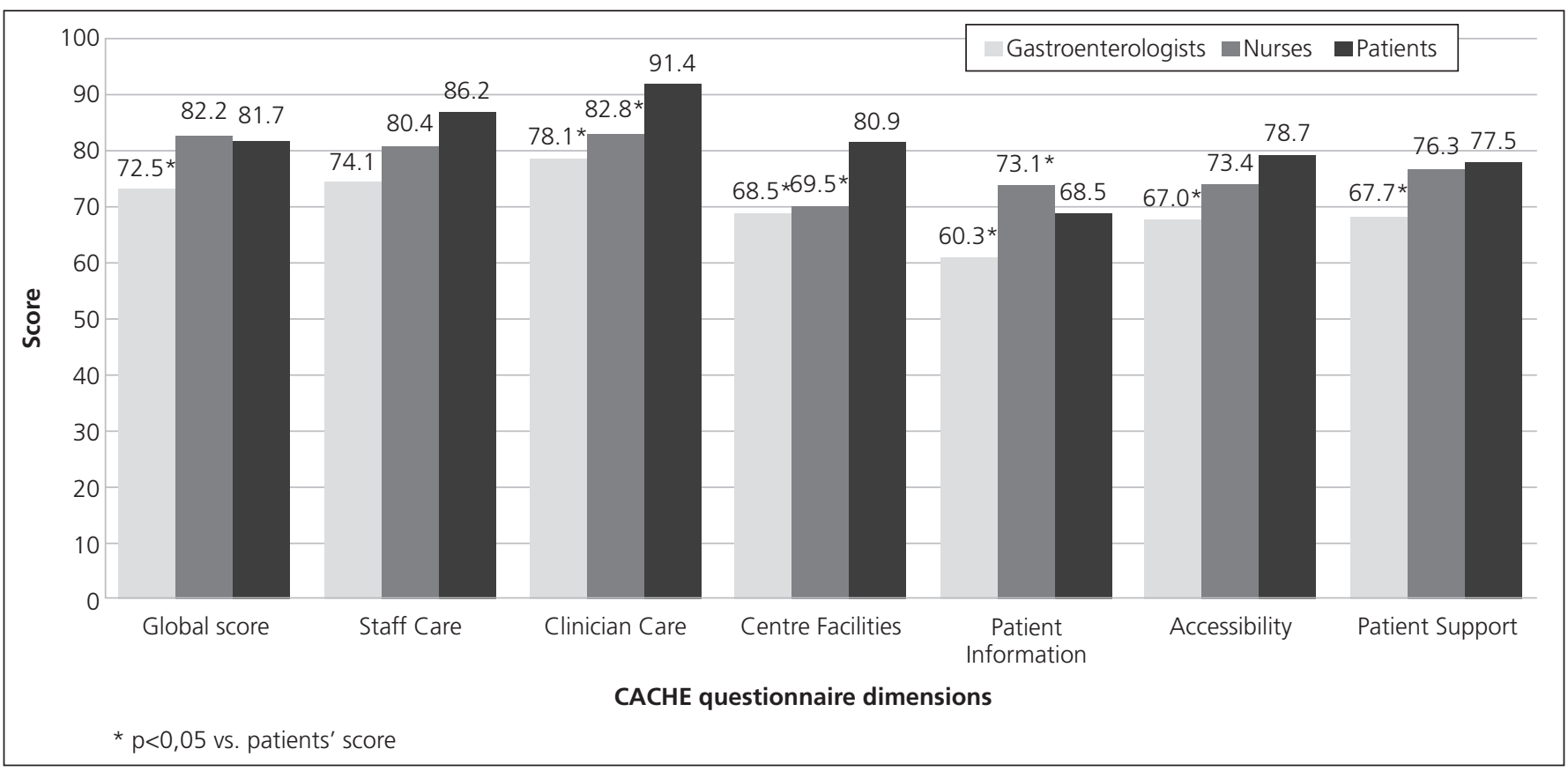

Fig. 2. CACHE questionnaire scores: Gastroenterologist and nurse perception of patient's satisfaction and patient (at baseline) assessment.

satisfaction, consequently the multiple regression analysis was not considered.

\section{DISCUSSION}

IBD is a chronic illness with an intense impact on patients' Health Related Quality of Life (HRQOL) and is associated with a high level of healthcare resource consumption $(6,7)$. Also the patient satisfaction with the healthcare services has been seen to be related to the clinical outcomes in patients with chronic illness (3). Taken all together, satisfaction with healthcare services might have a strong effect on patients and their HRQOL. This study has shown that IBD patients attending to Spanish hospital outpatients clinic for their health condition are highly satisfied with the healthcare services provided. Satisfaction is highest for features related to professional care (clinician and staff care domains).

When assessing UC patients in remission some discrepancies were found; at the baseline visit it was calculated that $66 \%$ of patients were in remission when using the Montreal classification but that $80 \%$ were in remission when the Mayo Clinic score was applied. These substantial differences might be explained for the different parameters and scores used for their calculation; these discrepancies were recently observed by Travis et al. (8).

The study found that the satisfaction with healthcare received among CD patients and UC patients is essentially the same. Considering the whole study sample, patient satisfaction does not change with respect to time and its score is not substantially affected by patient's sociodemo- graphic characteristics. Furthermore, patient satisfaction is not related to disease severity, length of duration, grade of disease control or if they are experiencing flare. Globally it can be considered that IBD patients are satisfied with the healthcare services provided independently of their health condition. Nonetheless, Kasman et al. found that female patients younger than 65 years old, white, with low income and having completed secondary school more frequently reported dissatisfaction with healthcare services (9). The differences between this and our study could be explained by the study population as Kasman reported results from a population based study including healthy people as well as ill patients for whom the use of healthcare resources might not be as high as it is for IBD patients (7).

The highest scores were seen on the two domains related to professional care, Clinician Care Satisfaction and Staff Care Satisfaction. Previously some studies in the United States found a high satisfaction level for patients with the healthcare services received $(10,11)$; one of these studies also analyzed the factors determining general satisfaction with medical services which were mainly the satisfaction with medical staff, reasonability of payment comfort and accessibility (9). IBD patients are frequent healthcare services users and they have frequent face-to-face contact with doctors and nurses. The study results are also in line with those from Jung el al. (9) as in Spain access to healthcare is universal any patient can ask to be assigned to another healthcare professional if they are not fully satisfied with their physician-patient relationship.

It has been seen that having a lasting patient-physician relationship is significantly associated with higher quality 
of relationship scores (12), an observation made in cancer survivors (13). This might be applied to our study patients too. Although we did not record the length of physician-patient relationship, IBD patients have had their disease for more an average of more than 8 years and they frequently visit their healthcare provider.

Some studies point out that not receiving healthcare when needed might not be due to accessibility issues, but rather to a patient's perception that their healthcare does not meet their individual needs (9). This statement is based on the fact the highest proportion of patients who reported dissatisfaction with the healthcare services were those with chronic disabling diseases for which there is no cure, such as fibromyalgia, $\mathrm{CD}$, chronic bronchitis/emphysema or migraine. The high patient satisfaction with Clinician and Staff Care might explain some of the differences between both studies as they might feel their needs are best covered through a long-term physician-patient relationship.

In the CACHE questionnaire, the Patient Information Satisfaction was the worst rated domain, indicating that a lack of information a key point for improvement. It has been previously seen in Spain that nearly three out of four IBD patients reported being satisfied with the quality of the healthcare received but that the information they received was inadequate (14). At present, patients suffering from gastrointestinal disorders identified some issues which were rated as being more important: the need for better explanations and more written information from clinicians, access to patient organisations and patient groups, consistency and coordination between GPs and hospital management and identifying disease-specific concerns and recommendations for defining outcomes that were relevant to patients (15). This lack of information is perceived not only by IBD patients, it has also been reported that almost a $60 \%$ of cancer survivors do not received enough information on health promotion or prevention topics (11). In general, it can be concluded that more information should be provided to patients, especially to those with chronic disabling or life threatening diseases in which the patient lifestyle is essential to preserve HRQOL.

Nurses' perception of IBD patient satisfaction with healthcare services is very close to that reported by patients. Nurses are known to have an essential role as healthcare providers in the management of patients with $\operatorname{IBD}(16,17)$, being usually involved on patients' follow-up and patients education on the most suitable lifestyle for their condition (18).

Gastroenterologists perceived patients' satisfaction on healthcare services to be less than that reported by patients, reporting lower scores than IBD patients for all the six domains. Gastroenterologists rated the six domains in the same order as patients. Patients and gastroenterologists agreed that Patient Information was the worst domain in terms of satisfaction. Some studies have reported coincident responses on physicians perceptions on patients feelings; a study with chronically ill adolescent patients showed that physician and patient responses were similar, but adolescent responses were less extreme (19). Similarly, Wilson et al. assessed the physician perception of patient's current health and they found moderate to good correlation between both criteria (20). Taking these data it can be assumed that both physicians and nurses accurately perceive patient perception of healthcare services received.

This study has some limitations. The study was not designed to assess differences between patient and healthcare professional perceptions of satisfaction, rather to validate the CACHE questionnaire and to study its psychometric properties. Moreover, the CACHE questionnaire for healthcare staff has not been validated, it was an adaptation of the CACHE patient's questionnaire. Some features may not have been included in the adaptation for healthcare staff.

Future investigations should address reasons why healthcare professionals perceive lower patient satisfaction with healthcare services than patients.

In conclusion, IBD patients in Spain are satisfied with the healthcare services provided; nurses' perception is similar to that of patients while physicians have a lower perception. Alignment of differences such as these between healthcare professional and patient perceptions will stimulate the use of new sources of information or other tools.

\section{ACKNOWLEDGMENTS}

This work was supported by MSD Spain. The sponsor had no involvement in the design, data collection, analysis, data interpretation and writing of the manuscript. We thank IMS Health that provided statistical and medical writing services. All authors have made substantial contributions to the conception and design of the study, or acquisition of data, or analysis and interpretation of data, drafting the article or revising it critically for important intellectual content, and final approval of the version to be submitted.

\section{REFERENCES}

1. Brullet E, Bonfill X, Urrutia G, Ruiz V, Cueto M, Clofent J, et al Epidemiological study on the incidence of inflammatory bowel disease in 4 Spanish areas. Spanish Group on the Epidemiological Study of Inflammatory Bowel Disease. Med Clin (Barc) 1998;110:651-6.

2. Vergara M, Casellas F, Badia X, Malagelada JR. Assessing the quality of life of household members of patients with inflammatory bowel disease: Development and validation of a specific questionnaire. Am J Gastroenterol 2002;97:1429-37.

3. Kaplan SH, Greenfield S, Ware JE Jr. Assessing the effects of physician-patient interactions on the outcomes of chronic disease. Med Care 1989;27(3 Supl.):S110-S127.

4. Pallis AG, Mouzas LA. Quality of health care in inflammatory bowel disease and its assessment. Annals of Gastroenterology 2002;15:143-7.

5. Casellas F, Ginard D, Vera I, Torrejón A on behalf of GETECCU. Development and testing of a new instrument to measure patient satisfaction with healthcare in inflammatory bowel disease: the CACHE questionnaire. Inflamm Bowel Dis 2013;19:559-68.

6. van der Have M, van der Aalst KS, Kaptein AA, Leenders M, Siersema PD, Oldenburg B, et al. Determinants of health-related quality of life 
in Crohn's disease: A systematic review and meta-analysis. J Crohns Colitis 2013 [Epub ahead of print].

7. Casellas F, López-Vivancos J, Vergara M, Malagelada J. Impact of inflammatory bowel disease on health-related quality of life. Dig Dis 1999;17:208-218.

8. Travis SP, Higgins PD, Orchard T, Van Der Woude CJ, Panaccione $\mathrm{R}$, Bitton A, et al. Defining remission in ulcerative colitis. Aliment Pharmacol Ther 2011;34:113-24.

9. Kasman NM, Badley EM. Beyond access: Who reports that healthcare is not being received when needed in a publicly-funded Healthcare System? Can J Public Health 2004;95:304-8.

10. Hojat M, Louis DZ, Maxwell K, Markham FW, Wender RC, Gonnella JS. A brief instrument to measure patients' overall satisfaction with primary care physicians. Fam Med 2011;43:412-7.

11. Jung M, Lee KH, Choi M. Perceived service quality among outpatients visiting hospitals and clinics and their willingness to re-utilize the same medical institutions. J Prev Med Public Health 2009;42:151-9.

12. Noyes R Jr, Kukoyi OA, Longley SL, Langbehn DR, Stuart SP. Effects of continuity of care and patient dispositional factors on the physician-patient relationship. Ann Clin Psychiatry 2011;23:180-5.

13. Arora NK, Reeve BB, Hays RD, Clauser SB, Oakley-Girvan I. Assessment of quality of cancer-related follow-up care from the cancer survivor's perspective. J Clin Oncol 2011;29:1280-9.
14. Casellas F, Fontanet G, Borruel N, Malagelada JR. The opinion of patients with IBD on healthcare received. Rev Esp Enferm Dig 2004:96:174-84.

15. Jones R, Hunt C, Stevens R, Dalrymple J, Driscoll R, Sleet S, et al. Management of common gastrointestinal disorders: quality criteria based on patients' views and practice guidelines. Br J Gen Pract 2009;59:e199-e208.

16. Nightingale AJ, Middleton W, Middleton SJ, Hunter JO. Evaluation of the effectiveness of a specialist nurse in the management of inflammatory bowel disease (IBD). Eur J Gastroenterol Hepatol 2000;12:967-73

17. Westwood N, Travis SP. Review article: What do patients with inflammatory bowel disease want for their clinical management? Aliment Pharmacol Ther 2008;27(Supl. 1):S1-S8.

18. Hernández-Sampelayo P, Seoane M, Oltra L, Marín L, Torrejón A, Vera MI, et al. Contribution of nurses to the quality of care in management of inflammatory bowel disease: a synthesis of the evidence. J Crohns Colitis 2010;4:611-22.

19. Britto MT, Slap GB, DeVellis RF, Hornung RW, Atherton HD, Knopf $\mathrm{JM}$, et al. Specialists understanding of the healthcare preferences of chronically ill adolescents. J Adolesc Health 2007;40:334-41.

20. Wilcox AR, Dragnev MC, Darcey CJ, Siegel CA. A new tool to measure the burden of Crohn's disease and its treatment: do patient and physician perceptions match? Inflamm Bowel Dis 2010;16:645-50. 